\title{
RESEARCH HIGHLIGHT Broken hearts: Iron overload, ferroptosis and cardiomyopathy
}

\author{
Marcus Conrad ${ }^{1}$ and Bettina Proneth ${ }^{1}$ \\ Cell Research (2019) 29:263-264; https://doi.org/10.1038/s41422-019-0150-y
}

\begin{abstract}
In a recently published paper in Proc Natl Acad Sci USA, Fang et al. elegantly demonstrated that doxorubicin treatment or transient ischemia/reperfusion in mice triggers ferroptosis, a distinctive form of necrotic cell death marked by irondependent lipid peroxidation, which is the main underlying mechanism in the pathogenesis of cardiomyopathy.
\end{abstract}

In 2016, heart disease was the leading cause of death for both men and women, accounting for over 600,000 cases annually in the United States alone. While the loss of terminally differentiated cardiomyocytes is a key event in the pathophysiological process of cardiac disorders, such as cardiomyopathies, the molecular mechanisms underlying cardiac cell death are incompletely understood.

Ferroptosis is a recently discovered, pharmacologically amenable form of regulated necrosis that is implicated in a myriad of pathophysiologically degenerative conditions including ischemia/ reperfusion injury (IRI), acute kidney failure, neurodegeneration and hemochromatosis. ${ }^{1,2}$ In terms of morphological, biochemical and genetic traits, ferroptosis is distinct from other cell death routines such as apoptosis, necroptosis or autophagy. One of the hallmarks of ferroptotic signaling is iron-dependent lipid peroxidation, which precedes the execution of cell death. Therefore, efforts to prevent ferroptosis in pathological contexts have focused on the use of radical trapping agents, such as Ferrostatin-1 (Fer-1) and Liproxstatin-1, as well as iron-chelating agents. $^{3,4}$

Doxorubicin (DOX) is a commonly used chemotherapeutic drug for the treatment of breast cancer, leukemia and other malignancies. Yet, the use of anthracyclines in the clinic is hampered by the occurrence of severe toxic side effects, particularly in cardiomyocytes, causing cardiomyopathy and heart failure. ${ }^{5}$ Therefore, Fang et al. ${ }^{6}$ further investigated the root cause of cardiomyocyte demise in response to systemic DOX exposure. In a mouse model of DOX-induced cardiomyopathy, they performed RNA-sequencing analysis to identify genes that are differentially expressed in the heart of treated versus control mice and found that heme oxygenase-1 (Hmox 1$)$ was significantly upregulated (Fig. 1). They could further show that activation of the Kelch-like ECH-associated protein 1 (Keap1)/nuclear factor (erythroid-derived 2)-like 2 (Nrf2) pathway through oxidative stress results in a strong upregulation of HMOX1. HMOX1, in turn, degrades heme, thereby causing the release of free cellular iron and subsequent cell death by iron overload with features reminiscent of ferroptosis. Of note, treatment of mice with the ferroptosis inhibitor Fer-1 robustly improved survival of DOX-treated animals, in stark contrast to necroptosis, autophagy and caspase inhibitors. The contribution of ferroptosis towards DOX-induced cardiomyopathy was further corroborated by the increase of the potential ferroptosis pharmacodynamics marker prostaglandin-endoperoxide synthase 2 (Ptgs2) mRNA, presence of distorted and strongly enlarged mitochondria with elevated levels of di- and trioxygenated phosphatidylethanolamines and significantly increased free nonheme iron in the heart. All these pathological features could be ameliorated by the use of Fer-1 and to a similar extent with Dexrazoxane, an FDA-approved drug with iron-chelating activity for the treatment of DOX-induced cardiotoxicity in cancer patients. In addition, both compounds showed tissue-protective effects in a mouse model of cardiac IRI, when administered 30 min prior to the ischemic insult to the heart.

In terms of the subcellular localization of free iron release and lipid peroxidation, the authors found significant accumulation of free iron and lipid peroxides in mitochondria compared to the cytosol in the hearts of DOX-treated animals. Additionally, administration of the mitochondria-targeted antioxidant mitoTEMPO yielded significant cardioprotective effects compared to its untargeted version (TEMPO). While the relevance of mitochondria in ferroptosis is currently still under debate, ${ }^{7,8}$ lipid peroxidation in the context of DOX-induced cardiotoxicity is likely confined to mitochondria.

Interestingly, although iron overload-induced ferroptosis seems to be a major contributor to the pathology of DOX-induced cardiomyopathy, blocking necroptosis in mice by knockout of receptor interacting serine/threonine kinase 3 (Ripk3) resulted in increased survival rates compared to Fer-1 treatment alone. This effect is consistent with previous observations demonstrating the simultaneous involvement of distinct cell death pathways in tissue damage. ${ }^{9}$ In fact, a recent study investigating acute kidney injury was able to show that regulated necrotic cell death events may proceed in a chronological order. ${ }^{10}$ Therefore, one may speculate whether iron overload-induced ferroptosis is the initial trigger for organ damage in DOX-induced cardiomyopathy followed by secondary necroptosis, presumably activated via necroinflammation. ${ }^{11}$

In summary, in their most recent paper, Fang et al. provide intriguing insights into the role of HMOX 1 as the major culprit for iron release in DOX-induced cardiotoxicity and draw for the first time an in vivo link between cardiac cell death and ferroptosis induced by iron overload. While the regulation of HMOX1 in ferroptosis is still considered pleiotropic as both protective and potentiating effects have been reported, ${ }^{12}$ it remains to be clarified whether distinct tissue and disease contexts are implicated.

Hence, the data presented by Fang et al. advocate for the development and exploitation of ferroptosis inhibitors as a new and promising therapeutic strategy to prevent cardiomyopathy induced by DOX, IRI and perhaps other insults. Future studies are thus warranted to explore the clinical implementation of these important findings for the treatment of heart disease. 


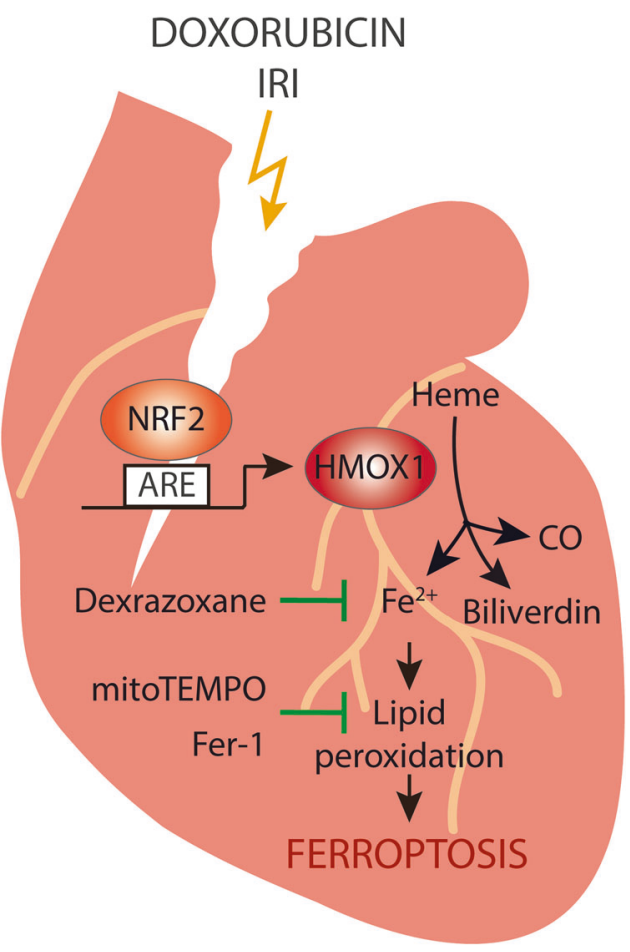

Fig. 1 Doxorubicin and ischemia/reperfusion injury (IRI) cause ferroptosis in cardiomyocytes and cardiomyopathy. Exposure of cardiac tissue to doxorubicin (or transient IRI) causes an NRF2dependent upregulation of HMOX1, heme degradation, iron overload and associated lipid peroxidation, the latter two are hallmarks of ferroptosis. The iron-chelating agent dexrazoxane, the lipid peroxidation inhibiting compounds mitoTEMPO and Fer-1 robustly improve tissue damage inflicted by doxorubicin and IRI, thus warranting the development of ferroptosis inhibitors for the effective treatement of heart disease. ARE antioxidant response element, Fer-1 ferrostatin-1, HMOX1 heme oxygenase-1, mitoTEMPO 2-(2,2,6,6-Tetramethylpiperidin-1-oxyl-4-ylamino)-2-oxoethyl) triphenylphosphonium chloride, NRF2 Nuclear factor (erythroid-derived 2)like 2

\section{ADDITIONAL INFORMATION}

Competing interests: The authors declare no competing interests.

\section{REFERENCES}

1. Conrad, M., Angeli, J. P., Vandenabeele, P. \& Stockwell, B. R. Nat. Rev. Drug Discov. 15, 348-366 (2016).

2. Stockwell, B. R. et al. Cell 171, 273-285 (2017).

3. Dixon, S. J. et al. Cell 149, 1060-1072 (2012).

4. Friedmann Angeli, J. P. et al. Nat. Cell Biol. 16, 1180-1191 (2014).

5. Singal, P. K. \& Iliskovic, N. N. Engl. J. Med. 339, 900-905 (1998).

6. Fang, X. et al. Proc Natl Acad Sci USA 116, 2672-2680 (2019).

7. Gao, M. et al. Mol. Cell 73, 354-363 (2019).

8. Gaschler, M. M. et al. ACS. Chem. Biol. 13, 1013-1020 (2018).

9. Linkermann, A. et al. Proc. Natl Acad. Sci. USA 111, 16836-16841 (2014).

10. Martin-Sanchez, D. et al. Proc. Natl Acad. Sci. USA 115, 4182-4187 (2018).

11. Proneth, B. \& Conrad, M. Cell Death Differ. 26, 14-24 (2019).

12. Chiang, S. K., Chen, S. E. \& Chang, L. C. Int. J. Mol. Sci. 20, E39 (2018). pii. 